\title{
Aplicación de nsqip-risk calculator en pacientes mayores de 80 años
}

\author{
Pamela Silva1', Dagoberto Ojeda'1, José Manuel Birimisa' ${ }^{1}$ Patricia Cisternas ${ }^{1}$
}

Introducción: Los octogenarios son un grupo emergente y de alto riesgo. La calculadora de riesgo ASC-NSQIP (American College of Surgeons-National Quality Improvement Program) es un modelo predictvo de morbimortalidad bastante preciso. Con la inclusión de esta calculadora en el algoritmo de evaluación del paciente sometido a cirugía no cardiaca de las guías de la American Heart Assosiation de 2014 se ha vuelto de nuestro interés su aplicación en la población mayor de 80 años.

Objetivos: Conocer la predicción de estadía hospitalaria y probabilidad de outcomes y compararlo con la estadía hospitalaria real y la frecuencia real de outcomes en pacientes mayores de 80 años sometida a cirugías electivas o de urgencia en nuestro centro utilizando la calculadora NSQIP-RC.

Material y Métodos: Trabajo observacional prospectivo. El tamaño muestral de 130 pacientes se calculó con sensibilidad de $90 \%$ y precisión $10 \%$, considerando prevalencia de complicaciones de $24,2 \%$. Después de la aprobación por el comité de ética se aplicó la calculadora de riesgo (http://riskcalculator. facs.org/) a pacientes quirúrgicos mayores de 80 años que fueran a cirugía electiva o urgencia entre diciembre de 2016 y diciembre de 2017. Se recolectó información demográfica, comorbilidades, ecocardiografía perioperatoria y se registró outcomes categorizados en ASC-NSQIP risk calculator (Complicaciones graves, cardiacas, Insuficiencia renal, Neumonia, Tromboembólicas y Mortalidad).

Resultados: Se obtuvieron 137 pacientes con edad promedio 85 años y $53 \%$ mujeres operados principalmente de cirugía traumatológica (29\%), digestivo/coloproctológica/hernias (13\%), urológica $12 \%$. Se encontró diferencia estadísticamente significativa en cuanto a estadía hospitalaria ( $p 0,0002$ ), del análisis multivariado resultó que la presencia de complicaciones graves aumentó 4 días la estadía postoperatoria (IC95\%: 1,6 - 10; $p=0,007$ ) y "otras cirugías" aumentó la estadía 3 días (IC95\%:1-7; p $0,031)$. No hubo diferencias estadísticamente significativas entre incidencias real y predicha de tromboembolismo pulmonar y mortalidad. Para complicaciones graves la sensibilidad de la calculadora es $79,44 \%$, la especificidad $59 \%$. Con un área bajo la curva ROC: 0,69; Intervalo de confianza 95\% $(0,61-0,77)$.

Conclusiones: Los pacientes quirúrgicos mayores de 80 años en nuestro centro tienen mayor estadía hospitalaria a la predicha por ASC-NSQIP risk calculator, del análisis de los resultados una de las causas podría ser la presencia de complicaciones graves que se asociaron a mayor estadía hospitalaria. Además, este modelo de riesgo quirúrgico subestimó la incidencia de complicaciones graves, cardiacas e Insuficiencia Renal y se correspondió con la incidencia de mortalidad y TEP.

\section{Referencias}

1. Fleisher $L A$, Fleischmann $K E$, Auerbach $A D$, Barnason SA, Beckman JA, Bozkurt B, et al.; American College of Cardiology; American Heart Association. 2014 ACC/AHA guideline on perioperative cardiovascular evaluation and management of patients undergoing noncardiac surgery: a report of the American College of Cardiology/American Heart Association Task Force on practice guidelines. J Am Coll Cardiol. 2014 Dec;64(22):e77-137.

2. Bilimoria KY, Liu Y, Paruch JL, Zhou L, Kmiecik $T E$, Ko CY, et al. Development and evaluation of the universal ACS NSQIP surgical risk calculator: a decision aid and informed consent tool for patients and surgeons. J Am Coll Surg. 2013 Nov;217(5):833-42.e1.

3. Bilimoria_KY, Liu Y, Paruch JL, Zhou L, Kmiecik TE, Ko CY, Cohen_ME. 